\title{
Frequency Estimation for Periodical Signal with Noise in Finite Time
}

\author{
Anton A. Pyrkin, Member, IEEE, Alexey A. Bobtsov, Senior Member, IEEE, \\ Denis Efimov, Member, IEEE, and Ali Zolghadri
}

\begin{abstract}
The frequency estimation technique with guaranteed finite time of convergence to a given accuracy of identification is presented. The approach for a high frequency noise rejection is proposed. The possibility of switching algorithm introduction for estimation quality improvement is discussed. The proposed solution has order three, that is smaller than in other existent solutions. Efficiency of the approach is demonstrated on examples of computer simulation.
\end{abstract}

Index Terms-adaptive observers, frequency estimation, disturbances rejection

\section{INTRODUCTION}

Many different approaches exist for adaptive identification of unknown sinusoid, see, for example, [1]-[10], [12]-[14], [22]. Some of these approaches are not restricted to the case of a single sinusoid, in particular, a biased sinusoidal signal is considered in [3], [9], [17], [19], [20], and the case of multiple sinusoids with different frequencies is presented in [22].

In spite of diversity solutions obtained so far, the problem of reliable frequency estimation in the presence of noisy measurements and in a finite time has not a comprehensive solution. The estimation accuracy and the time of convergence are the most important characteristics of an adaptive algorithm for practical implementation. In the present work we will address the issues of convergence time and robustness for a frequency estimator.

Our approach is based on the ideas introduced in [3]- [7], [15]. The dynamic order of the adaptive law is equal to three, which compares favorably with known results [9], [13], [16], [22]. In [2] a similar algorithm is proposed but in this paper the adaptive law is simpler requiring less calculations. The finite time of frequency estimate convergence to a predefined accuracy is evaluated. It is shown that this time can be assigned by tuning the algorithm parameters. Finally, a switching algorithm is proposed that is able to filter the measurement noise ensuring the required minimal accuracy in a finite time and zero estimation error asymptotically.

This work was supported by the Federal Target Program "R\&D in Priority Areas of Russia's Scientific and Technological Development in 2007-2013" (project 11.519.11.4007).

A. Bobtsov and A. Pyrkin are with the Department of Control Systems and Informatics, Saint Petersburg State University of Information Technologies Mechanics and Optics, Kronverkskiy av. 49, Saint Petersburg, 197101, Russia. E-mail: a.pyrkin@gmail.com, bobtsov@mail.ru.

D. Efimov and A. Zolghadri are with IMS-LAPS, University Bordeaux 1, 351 cous de la Liberation, Talence, Bordeaux, France. E-mail: denis.efimov@ims-bordeaux.fr, ali.zolghadri@ims-bordeaux.fr.

A. Bobtsov is with the Laboratory "Control of Complex Systems", Institute for Problems of Mechanical Engineering, Bolshoy pr. V.O. 61, St.Petersburg, 199178, Russia.
The problem formulation is introduced in Section 2. The biased signal estimation without noise is presented in Section 3. The case of noisy measurements is treated in Section 5. The hint on the asymptotic estimation error improvement is given in Section 6. The switching algorithm is developed in Section 7. The results of computer simulation of the proposed algorithms are included confirming efficiency of the approach.

\section{PROBLEM FORMULATION}

Consider the periodical signal $y(t)$ corrupted by noise

$$
y(t)=\sigma+\mu \sin (\omega t+\phi)+\delta(t),
$$

where $\omega \in \mathbb{R}_{+}$is the frequency of signal, $\sigma \in \mathbb{R}$ is the bias or offset, $\mu \in \mathbb{R}_{+}$is the amplitude, $\phi \in \mathbb{R}$ is the phase shift, $\delta: \mathbb{R}_{+} \rightarrow \mathbb{R}$ is the high-frequency noise term. The following assumptions are used in the paper.

A1 A lower bounds on the signal frequency $\omega_{0} \in \mathbb{R}_{+}$ and amplitude $\mu_{0} \in \mathbb{R}_{+}$are known, i.e. $\omega \geq \omega_{0}$ and $\mu \geq \mu_{0}$.

A2 The amplitude of the signal is greater than the maximum value of the irregular term: $\mu>\sup _{t \geq 0}|\delta(t)|$.

The objective is to find the frequency $\omega(t)$ in a finite time with desired accuracy

$$
|y(t)| \leq \Delta, \forall t \geq t_{0},
$$

where $\Delta \in \mathbb{R}_{+}$is the desired value of estimation error and $t_{0} \in \mathbb{R}_{+}$is the transient time.

\section{BiASED SinUsoidAL Signal}

To derive the main result let us consider the signal $y(t)$ without term $\delta(t)$. Following the idea presented in ( [5], [6]) we start by introducing the linear second-order filter

$$
\xi(s)=\frac{\lambda_{0}^{2}}{\gamma(s)} y(s),
$$

where $\lambda_{0}>0, \gamma(s)=s^{2}+\gamma_{1} s+\gamma_{0}$ is a Hurwitz polynomial with two different eigenvalues $\lambda_{1}$ and $\lambda_{2}$. Let $\gamma_{0}=\lambda_{0}^{2}$ and $\lambda=\min _{i=1,2}\left\{\left|\operatorname{Re} \lambda_{i}\right|\right\}$.

Lemma 1. For the filter (3) and the input signal

$$
y(t)=\sigma+\mu \sin (\omega t+\phi)
$$

the relation

$$
\dddot{\xi}(t)=\theta \dot{\xi}(t)+\varepsilon(t)
$$


holds, where functions $\dot{\xi}(t)$ and $\dddot{\xi}(t)$ are derivatives of the output variable of the linear filter (3)

$$
\dot{\xi}(s)=\frac{\lambda_{0}^{2} s}{\gamma(s)} y(s), \quad \dddot{\xi}(s)=\frac{\lambda_{0}^{2} s^{3}}{\gamma(s)} y(s),
$$

$\theta=-\omega^{2}$, function $|\varepsilon(t)| \leq \rho_{0} e^{-\lambda t}$ and its derivatives are bounded by an exponentially decaying function.

Proof: It is well known [3], [21] that signal $y(t)=\sigma+$ $\mu \sin (\omega t+\phi)$ can be generated by the system

$$
\dddot{y}(t)+\omega^{2} \dot{y}(t)=0 .
$$

Taking the Laplace transformation in (7) we obtain

$$
s^{3} y(s)=\theta s y(s)+D(s),
$$

where $D(s)$ denotes initial conditions. Multiplying (8) on $\frac{\lambda_{0}^{2}}{\gamma(s)}$ with respect to (3) yields

$$
s^{3} \xi(s)=\theta s \xi(s)+\frac{\lambda_{0}^{2}}{\gamma(s)} D(s) .
$$

After inverse Laplace transformation in (9) we have necessary equation (5)

$$
\dddot{\xi}(t)=\theta \dot{\xi}(t)+\varepsilon(t)
$$

where $\varepsilon(t)=\mathcal{L}^{-1}\left\{\frac{\lambda_{0}^{2} D(s)}{\gamma(s)}\right\}$. By force the polynomial $\gamma(s)$ the function $\varepsilon(t)$ can be represented as a sum of decaying exponents. Therefore, derivatives of these functions are also exponentially decaying.

The adaptive scheme for frequency estimation is presented in the following theorem.

Theorem 2. The update law

$$
\begin{aligned}
& \hat{\omega}=\sqrt{|\hat{\theta}|}, \\
& \hat{\theta}=\chi+k \dot{\xi} \ddot{\xi}, \\
& \dot{\chi}=-k \dot{\xi}^{2} \hat{\theta}-k \ddot{\xi}^{2},
\end{aligned}
$$

where $k>0$, guarantees that the estimation error $\tilde{\omega}=\omega-\hat{\omega}$ exponentially converges to zero:

$$
|\tilde{\omega}(t)| \leq \rho_{1} e^{-\beta_{1} t}, \quad \rho_{1}, \beta_{1}>0, \quad \forall t \geq 0 .
$$

Proof: Using Lemma 1, we compute the derivative of the estimation error $\tilde{\theta}=\theta-\hat{\theta}$ :

$$
\begin{aligned}
\dot{\tilde{\theta}}(t) & =\dot{\theta}-\dot{\hat{\theta}}(t) \\
& =-\dot{\chi}(t)-k \ddot{\xi}^{2}(t)-k \dot{\xi}(t) \dddot{\xi}(t) \\
& =k \dot{\xi}^{2}(t) \hat{\theta}(t)+k \ddot{\xi}^{2}(t)-k \ddot{\xi}^{2}(t)-k \dot{\xi}(t) \dddot{\xi}(t) \\
& =k \dot{\xi}^{2}(t) \hat{\theta}(t)-k \dot{\xi}^{2}(t) \theta-k \dot{\xi}(t) \varepsilon(t) \\
& =-k \dot{\xi}^{2}(t) \tilde{\theta}(t)-k \dot{\xi}(t) \varepsilon(t) .
\end{aligned}
$$

Consider the Lyapunov function

$$
V(t)=\frac{1}{2} f(t) \tilde{\theta}^{2}(t),
$$

where $f(t)$ is a bounded positive function that will be chosen later. We get

$$
\begin{aligned}
\dot{V} & =\frac{1}{2} \dot{f} \tilde{\theta}^{2}+f \tilde{\theta} \dot{\tilde{\theta}} \\
& =\frac{1}{2} \dot{f} \tilde{\theta}^{2}-k f \dot{\xi}^{2} \tilde{\theta}^{2}-k f \dot{\xi} \tilde{\theta} \varepsilon \\
& \leq \frac{1}{2} \dot{f} \tilde{\theta}^{2}-\frac{k}{2} f \dot{\xi}^{2} \tilde{\theta}^{2}+\frac{k}{2} f \varepsilon^{2} .
\end{aligned}
$$

From (10) we have

$$
\dot{\xi}(t)=\mu_{1} \sin \left(\omega t+\phi_{1}\right)+\varepsilon_{1}(t),
$$

where $\mu_{1}$ is an amplitude, $\phi_{1}$ is a phase shift, $\varepsilon_{1}(t)$ is an exponentially decaying function as well as its derivatives. From (18) we obtain

$$
\begin{aligned}
\dot{\xi}^{2}(t)= & \left(\mu_{1} \sin \left(\omega t+\phi_{1}\right)+\varepsilon_{1}(t)\right)^{2} \\
= & \frac{1}{2} \mu_{1}^{2}-\frac{1}{2} \mu_{1}^{2} \cos \left(2 \omega t+2 \phi_{1}\right) \\
& +2 \mu_{1} \sin \left(\omega t+\phi_{1}\right) \varepsilon_{1}(t)+\varepsilon_{1}^{2}(t) .
\end{aligned}
$$

Substituting (19) into (17) we get

$$
\begin{aligned}
\dot{V} \leq & \frac{1}{2} \dot{f} \tilde{\theta}^{2}-\frac{1}{2} k \mu_{1}^{2} \frac{1}{2} f \tilde{\theta}^{2} \\
& -\frac{1}{2} k f \tilde{\theta}^{2}\left(-\frac{1}{2} \mu_{1}^{2} \cos \left(2 \omega t+2 \phi_{1}\right)\right. \\
& \left.+2 \mu_{1} \sin \left(\omega t+\phi_{1}\right) \varepsilon_{1}+\varepsilon_{1}^{2}\right)+\frac{1}{2} k f \varepsilon^{2} .
\end{aligned}
$$

Let $f(t)=e^{g(t)}$, where

$$
\begin{aligned}
g(t)= & k \int_{0}^{t}\left(2 \mu_{1} \sin \left(\omega \tau+\phi_{1}\right) \varepsilon_{1}(\tau)+\varepsilon_{1}^{2}(\tau)\right) d \tau \\
& -\frac{1}{4} k \mu_{1}^{2}\left(\sin \left(2 \omega t+2 \phi_{1}\right)-\sin \left(2 \phi_{1}\right)\right) .
\end{aligned}
$$

Since $g(t)$ depends on bounded harmonic and exponential functions, it is bounded, $|g(t)| \leq C_{1}$, where $C_{1}$ is a positive constant. Therefore, $e^{-C_{1}} \leq f(t) \leq e^{C_{1}}$ and the Lyapunov function $V$ is well defined.

Using (21), from (20) after a number of simple transformation we obtain

$$
\begin{aligned}
\dot{V}(t) & \leq-\frac{1}{2} k \mu_{1}^{2} \frac{1}{2} f(t) \tilde{\theta}^{2}(t)+\frac{1}{2} k f(t) \varepsilon^{2}(t) \\
& \leq-C_{2} V(t)+\rho_{2} e^{-\beta_{2} t}
\end{aligned}
$$

where $C_{2}=\frac{1}{2} k \mu_{1}^{2}, \rho_{2}=\frac{1}{2} k e^{C_{1}} \rho_{0}^{2}$, and $\beta_{2}=2 \lambda$ are positive constants. Using comparison principle [11], it is easy to show that

$$
V(t) \leq \rho_{3} e^{-\beta_{3} t}
$$

where $\rho_{3}>0$ and $\beta_{3}=\min \left\{C_{2}, \beta_{2}\right\}$. From (16) and (23) we get

$$
|\tilde{\theta}(t)| \leq \sqrt{\frac{2 \rho_{3}}{e^{-C_{1}}}} e^{-\frac{1}{2} \beta_{3} t} .
$$

Using (11), it is straightforward to show that

$$
|\tilde{\omega}(t)| \leq \sqrt{|\tilde{\theta}(t)|} \leq \rho_{1} e^{-\beta_{1} t}
$$

where $\rho_{1}=\sqrt[4]{2 \rho_{3} e^{C_{1}}}$ and $\beta_{1}=\beta_{3} / 4$. 
Remark 3. From (23) follows that transients time for the variable $V(t)$ explicitly depends on $k$ and $\lambda$ via parameter $\beta_{3}$. The increasing $k$ and $\lambda$ means acceleration of convergence $V(t)$ to zero as well as convergence of the estimate $\hat{\omega}$ to the real value $\omega$.

Example 4. Consider the numerical example to demonstrate how the adaptive scheme (11)-(13) works. Let the filter (3) looks like

$$
\xi(s)=\frac{2 \lambda^{2}}{s^{2}+3 \lambda s+2 \lambda^{2}} y(s),
$$

where denominator has the eigenvalues $\lambda_{1}=-\lambda$ and $\lambda_{2}=$ $-2 \lambda$.

In Fig. 1 the frequency estimates are shown for different values of $k$ and $\lambda$ and the signal $y(t)=3+4 \sin (2 t+1)$.

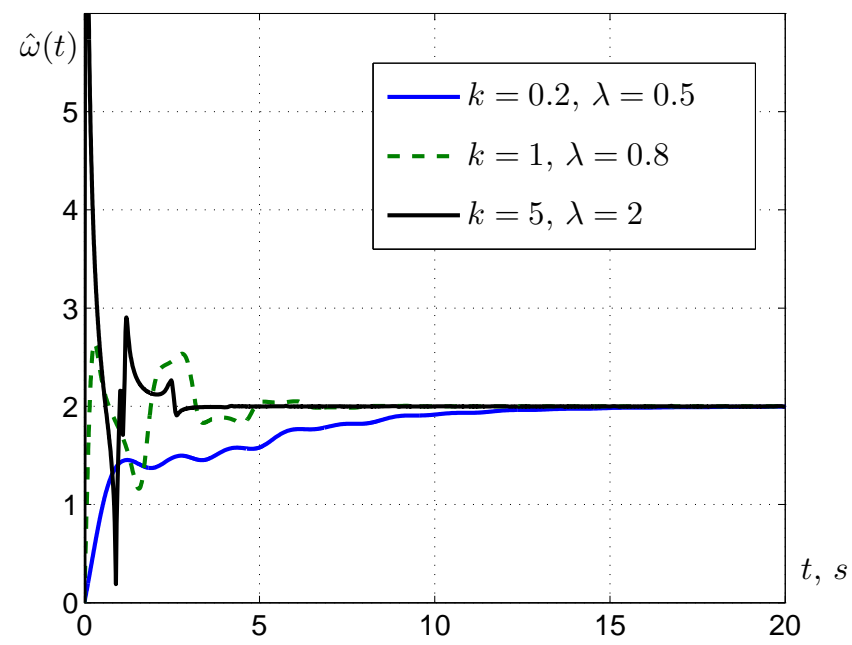

Figure 1: Estimates for the frequency $\omega=2$.

\section{Periodical Signal With Noise}

In this section we consider the signal $y(t)$ corrupted by noise $\delta(t)$. For signal (1) we will make the similar analysis as in the previous section.

The signal (1) is the output of the system

$$
\dddot{y}(t)+\omega^{2} \dot{y}(t)=\delta_{0}(t),
$$

where $\delta_{0}$ is the exciting noise. For (27) we have

$$
\dddot{\xi}(t)=\theta \dot{\xi}(t)+\varepsilon(t)+\delta_{\xi}(t)
$$

where $\delta_{\xi}(t)=\frac{\lambda_{0}^{2}}{\gamma(p)} \delta_{0}(t)$. Analyzing the adaptive law (11)(13) and the Lyapunov function (16) with respect to (1), (3) and (28) it is straightforward though lengthy to show for (22) and (23)

$$
\dot{V}(t) \leq-\beta_{3} V(t)+k \delta_{V}(t),
$$

where $\delta_{V}(t)$ is an irregular term caused by $\delta_{\xi}(t), \varepsilon(t)$ and the parameter $\beta_{3}$ dependent on $k$ and $\lambda$ is defined in (23). Then we get for (25)

$$
|\tilde{\omega}(t)| \leq \rho_{1} e^{-\beta_{1} t}+C_{\omega}
$$

where $C_{\omega}$ is a positive constant that depends on amplitude of the irregular term in the signal $y(t)$.

Example 5. Consider the next example to show robust properties of the adaptive law (11)-(13). In figures $3 a-3 c$ the frequency estimates are shown for different values of $k$ and $\lambda$ and the signals $y(t)$ from the figures $2 \mathrm{a}-2 \mathrm{c}$.

\section{Estimation QuAlity ANALYsis}

In this section we find the relation between parameters $k$ and $\lambda$ and the asymptotic accuracy of the frequency estimate $\hat{\omega}(t)$, i.e. the maximum value of the estimation error $|\tilde{\omega}(t)|$.

Consider the inequality (29) and auxiliary system

$$
\dot{x}(t)=-\beta_{3} x(t)+k \delta_{V}(t) .
$$

Rewrite the system (31) as follows

$$
x(t)=\left[\frac{k}{p+\beta_{3}}\right] \delta_{V}(t),
$$

where $p=d / d t$ is the differentiation operator. To analyze asymptotic behavior of the low-pass filter

$$
W(p)=\frac{k}{p+\beta_{3}},
$$

we will use the magnitude Bode plot of (33). For a pure harmonic disturbance $\delta_{V}$ with the frequency spectrum starting from the frequency $\omega_{\delta} \gg \omega$, the asymptotic amplitude of $x$ is proportional to the value

$$
\left|W\left(j \omega_{\delta}\right)\right|=k / \sqrt{\omega_{\delta}^{2}+\beta_{3}^{2}},
$$

that in its turn is dependent on parameters $k$ and $\lambda\left(\beta_{3}=\right.$ $\min \left\{0.5 k \mu_{1}^{2}, 2 \lambda\right\}$ from (23)). Therefore, the asymptotic magnitude of $x$ decreases if $k$ and $\lambda$ become smaller, especially for the high-frequency terms in $\delta_{V}(t)$ :

$$
\lim _{k \rightarrow 0, \lambda \rightarrow 0}\left|W\left(j \omega_{\delta}\right)\right|=\lim _{k \rightarrow 0, \lambda \rightarrow 0} \frac{k}{\omega_{\delta}}=0 .
$$

Using comparison principal [11] we find from (29) and (31) that, if $x(0) \geq V(0)$, then

$$
V(t) \leq x(t) .
$$

Thus the parameters $k$ and $\lambda$ regulate the asymptotic convergence of $V(t)$, as well as $\tilde{\omega}(t)$, to a neighborhood of the origin.

It is easy to see that the transient time for $V(t)$ is defined by the time constant $\beta_{3}$. It is well-known that the exponential function is converged over three time constants in the $5 \%$ zone of its initial amplitude (i.e. for any $b \in \mathbb{R}_{+}, b e^{-\beta_{3} t} \leq 0.05 b$ for all $t \geq 3 / \beta_{3}$ ). So we have the time

$$
t_{f}=\frac{3}{\beta_{3}},
$$

after which the function $\hat{\omega}(t)$ is converged to a bounded domain around the ideal value $\omega$. It is possible to say that the constant $t_{f}$ defines the time window length after which the initial conditions influence on the transients can be neglected. For a high-frequency noise the asymptotic accuracy of estimation is governed by the expression (34). 


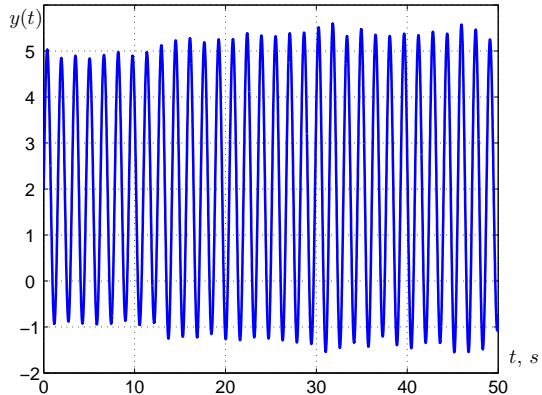

(a) Transients for $y(t)=2+3 \sin (4 t)+\delta(t)$, where $\delta(t)$ is the bounded noise.

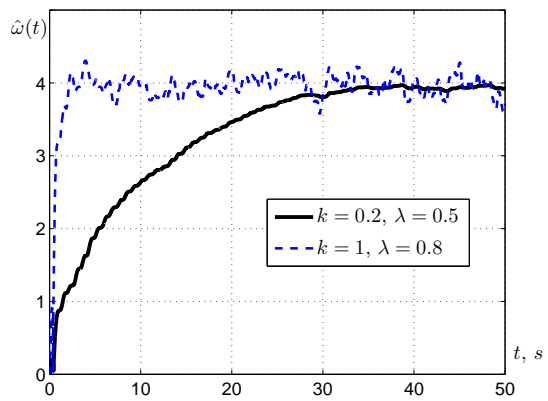

(a) Estimates for the frequency $\omega=4$ of the signal $2 \mathrm{a}$.

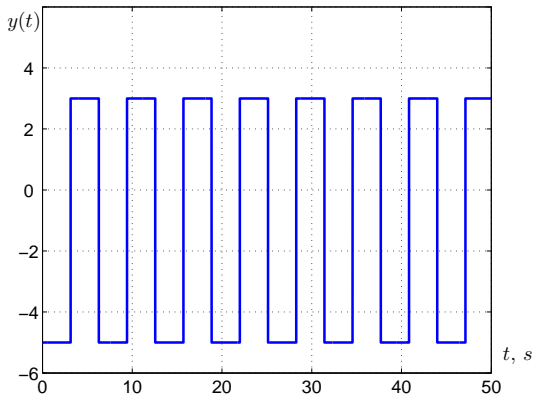

(b) Transients for "meander" signal.

Figure 2: Transients for $y(t)$.

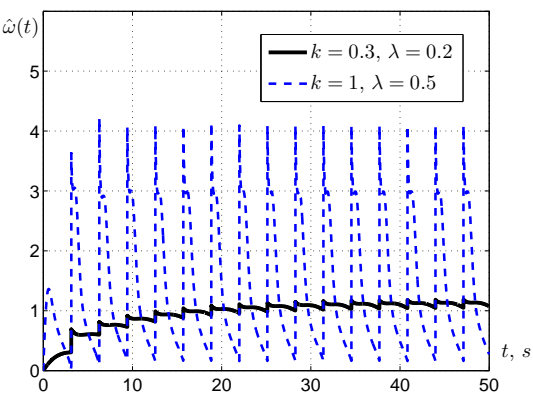

(b) Estimates for the frequency of the "meander" signal $2 \mathrm{~b}$.

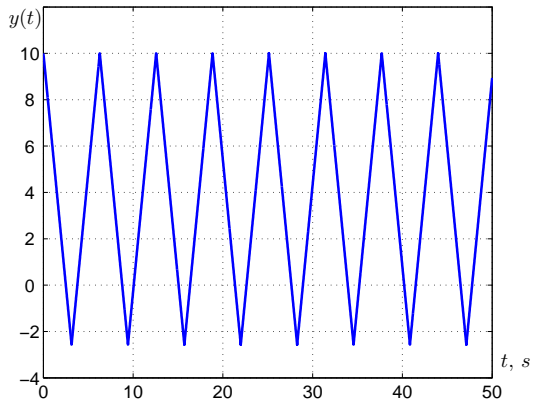

(c) Transients for "triangle" signal.

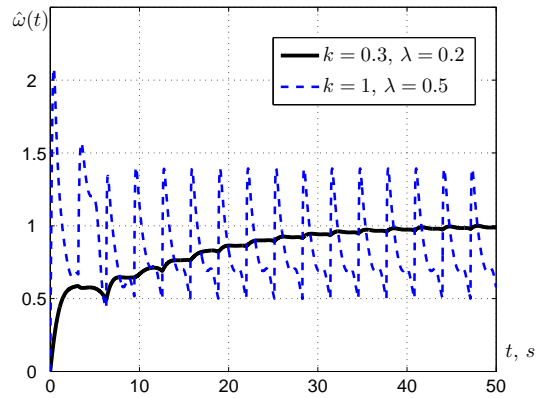

(c) Estimates for the frequency of the "triangle" signal $2 \mathrm{c}$.

Figure 3: Transients for frequency estimates $\hat{\omega}(t)$.

\section{HYBRID SWITCHING}

In this section a hybrid scheme of switching for the parameters $k$ and $\lambda$ is presented, that allows us to improve the asymptotic accuracy of estimation of $\omega$ by the variable $\hat{\omega}$. Moreover, for a pure high-frequency noise, the estimation error goes to zero with time growth.

Let $t_{i}, i \geq 0$ with $t_{0}=0$ be an increasing sequence of the switching time instants, define $k_{i}=k\left(t_{i}\right)$ and $\lambda_{i}=\lambda\left(t_{i}\right)$, then:

$$
\begin{gathered}
k_{i+1}=\frac{1}{\alpha} k_{i}, \quad \lambda_{i+1}=\frac{1}{\alpha} \lambda_{i}, \\
t_{i+1}=t_{i}+3 / \beta_{3}\left(k_{i}, \lambda_{i}\right), \\
k(t)=k_{i}, \lambda(t)=\lambda_{i} \quad \forall t \in\left(t_{i}, t_{i+1}\right),
\end{gathered}
$$

where $\alpha>1$. The function $\beta_{3}$ explicitly depends on $k$ and $\lambda$, moreover this dependence is linear and

$$
\beta_{3}\left(k_{i+1}, \lambda_{i+1}\right)=\frac{1}{\alpha} \beta_{3}\left(k_{i}, \lambda_{i}\right) .
$$

So we can rewrite (38) in a simpler form:

$$
\begin{aligned}
t_{i+1} & =t_{i}+\Delta t_{i}, \quad t(0)=0 \\
\Delta t_{i+1} & =\alpha \Delta t_{i}, \Delta t_{0}=\frac{3}{\beta_{3}\left(k_{0}, \lambda_{0}\right)} .
\end{aligned}
$$

Thus using the algorithm (37), (39), (40) it is possible to ensure the fast initial convergence of the error $\tilde{\omega}$ to some vicinity of the origin. Next, decreasing the values of $k_{i}$ and $\lambda_{i}$ we increase the settling time interval $\Delta t_{i}$, but we may guarantee the term $\left|W\left(j \omega_{\delta}\right)\right|$ convergence to zero in asymptotic. This fact is equivalent to high-frequency noise filtering by the switching algorithm. Note, that it is not possible to filter the initial measured signal $y$ for the noise $\delta$ cancellation, since the useful frequency $\omega$ and the noise frequency $\omega_{\delta}$ are not known a priori.

Example 6. Let $y(t)=2+8 \sin (1.6 t+1)+\delta$ (the signal is shown in Fig. 4a), where $\delta$ is a white noise with restricted spectrum and power 0.1 (presented separately in Fig. 4b). The results of the frequency estimation by the proposed algorithm with the fixed parameters $(k=0.5$ and $\lambda=0.3)$ and the switching one $\left(\Delta t_{0}=10, \alpha=1.25, k_{0}=0.5, \lambda_{0}=0.3\right)$ are plotted in Fig. 4c. The parameter adjustment by the switching algorithm is shown in Fig. 4d. As it possible to conclude from these results, the switching algorithm has the same initial time of convergence to a vicinity of the identifying value, while asymptotically it rejects the measurement noise influence on the estimation error. These results confirm the theoretical findings of the work.

\section{CONCLUSION}

The problem of frequency estimation for a noisy and biased harmonic signal is studied. The estimation algorithm of the third order is proposed and the time of convergence and accuracy of the algorithm are evaluated. The switching algorithm is developed that allows us to cancel the asymptotic influence of a pure harmonic and high-frequency noise on the estimation error. The efficiency is illustrated by simulated examples. 


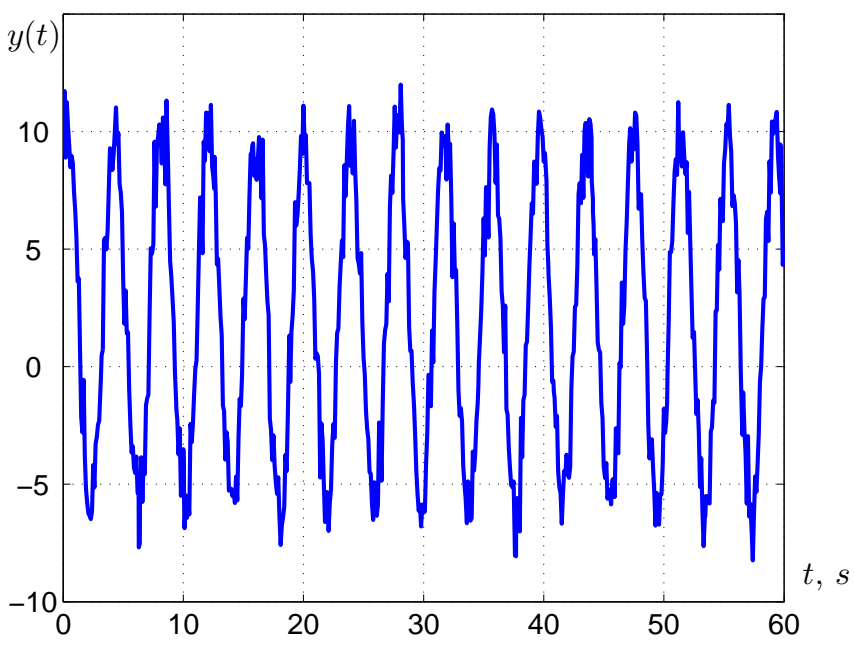

(a) Transients for $y(t)$.

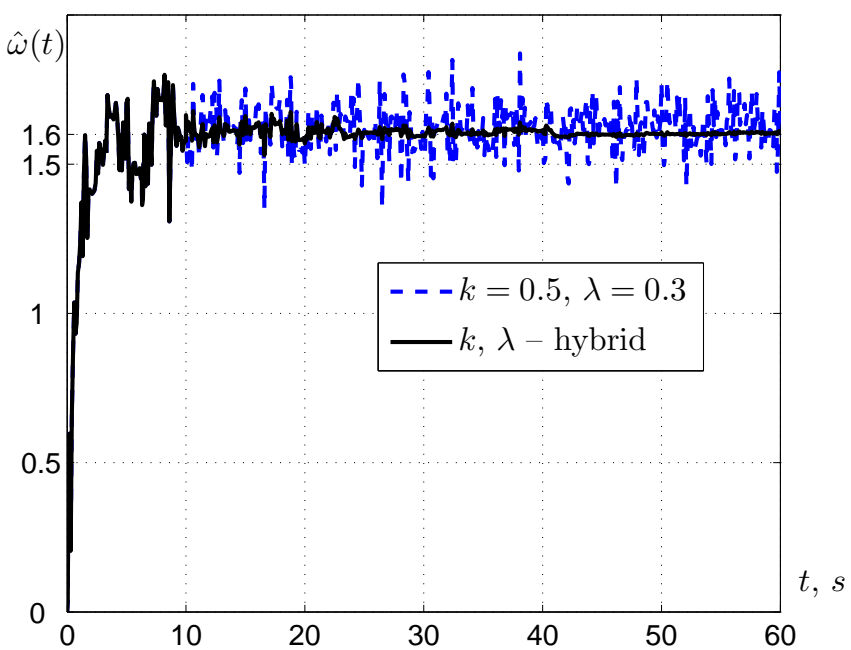

(c) Estimates for the frequency with time-invariant and hybrid switching parameters $k$ and $\lambda$.

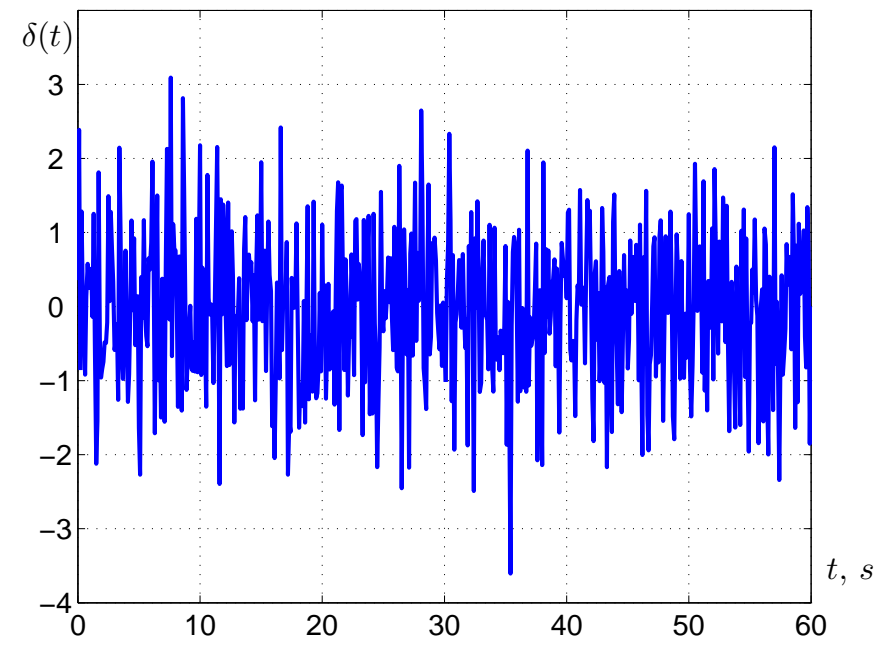

(b) The measurement noise.

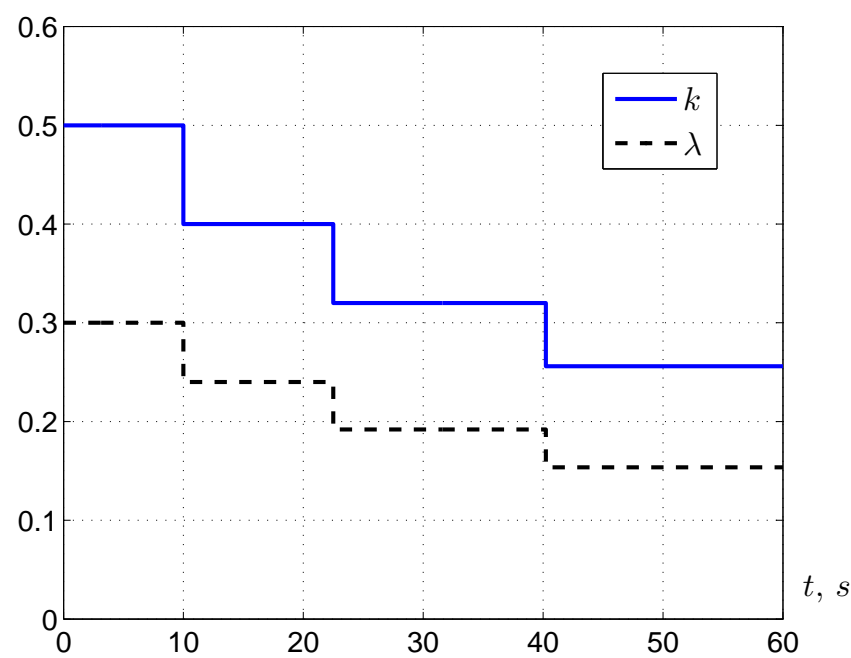

(d) Transients for hybrid switching of the parameters $k$ and $\lambda$, where $k(0)=$ $0.5, \lambda(0)=0.3, \Delta t(0)=10$, and $\alpha=1.25$.

Figure 4: Transients for hybrid switching parameters $k$ and $\lambda$.

\section{REFERENCES}

[1] S. V. Aranovskii, A. A. Bobtsov, A. A. Pyrkin, "Adaptive observer of an unknown sinusoidal output disturbance for linear plants ," Automation and Remote Control, vol. 70, N. 11, pp. 1862-1870, 2009.

[2] S. Aranovskiy, A. Bobtsov, A. Kremlev, N. Nikolaev, O. Slita, "Identification of frequency of biased harmonic signal" European Journal of Control, N. 2, pp. 129-139, 2010.

[3] A. A. Bobtsov, "Output control algorithm with the compensation of biased harmonic disturbances," Automation and Remote Control, vol. 69, N. 8, pp. 1289-1296, 2008.

[4] A. Bobtsov, New approach to the problem of globally convergent frequency estimator, Int. Journal of Adaptive Control and Signal Processing, 2008, N. 3, pp. 306-317.

[5] A. A. Bobtsov, A. A. Pyrkin, "The compensation of a harmonic perturbation under conditions of a delay in control," Journal of Computer and System Sciences International, vol. 47., N. 4, pp. 513-518, 2008.

[6] A. A. Bobtsov, A. A. Pyrkin, "The compensation of a harmonic disturbance for linear plant with any relative degree," Automation and Remote Control, vol. 70, N. 3, pp. 449-456, 2009.

[7] A. Bobtsov, S. Kolyubin, A. Pyrkin, "Compensation of Unknown Multi-harmonic Disturbances in Nonlinear Plant with Delayed Control," Automation and Remote Control, 2010, N. 11, 2383-2394.

[8] M. Bodson, S. C. Douglas, "Adaptive algorithms for the rejection of periodic disturbances with unknown frequencies," Automatica, vol. 33, pp. 2213-2221, 1997. plant parameters," Automatica, vol. 45, 2074-2081.

[9] M. Hou, "Amplitude and frequency estimator of a sinusoid," IEEE Trans. on Automatic Contr., vol. 50, 855-858.

[10] L. Hsu, R. Ortega, and G. Damm, "A globally convergent frequency estimator", IEEE Transactions on Automatic Control, vol. 44, 698-7139.

[11] H. Khalil, Nonlinear Systems, third edition, Upper Saddle River, New Jersey: Prentice Hall, 2002.

[12] R. Marino, P. Tomei, "Global Estimation of Unknown Frequencies," IEEE Transactions on Automatic Control, vol. 47, pp. 1324-1328, 2002.

[13] R. Marino, G. L. Santosuosso, P. Tomei, "Robust adaptive compensation of biased sinusoidal disturbances with unknown frequency," Automatica, vol. 39, pp. 1755-1761, 2003.

[14] R. Marino, G. L. Santosuosso, P. Tomei, "Regulation of Linear Systems with Unknown Additive Sinusoidal Sensor Disturbances," in Proc. 17th IFAC World Congress, Seoul, 2008, pp. 4102-4107.

[15] V. O. Nikiforov, "Adaptive non-linear tracking with complete compensation of unknown disturbances," European Journal of Control, vol. 4 N. 2, pp. 132-139, 1998

[16] A. W. Olbrot, "Stabilizability, detectability, and spectrum assignment for linear autonomous systems with general time delays," IEEE Trans. Autom. Control, vol. 23, pp. 887-890, 1978.

[17] A. Pyrkin, A. Smyshlyaev, N. Bekiaris-Liberis, M. Krstic Rejection of Sinusoidal Disturbance of Unknown Frequency for Linear System with Input Delay. American Control Conference. Baltimore, USA, 2010.

[18] A. Pyrkin, A. Smyshlyaev, N. Bekiaris-Liberis, M. Krstic Output Control Algorithm for Unstable Plant with Input Delay and Cancellation of Un- 
known Biased Harmonic Disturbance. Time Delay System Conference, Prague, Czech Republic, 2010.

[19] A.A. Pyrkin, A.A. Bobtsov, A.S. Kremlev, Rejection of unknown biased harmonic disturbance for nonlinear system with input delay. 9th IFAC Workshop on Time Delay System, Prague, Czech Republic, 2010.

[20] A. Pyrkin, A. Bobtsov, S. Chepinskiy, and Y. Kapitanyuk Compensation of Unknown Multiharmonic Disturbance for Nonlinear Plant with Delay in Control. 8th IFAC Symposium on Nonlinear Control Systems, Bologna, Italy, 2010.

[21] A. Pyrkin, The adaptive compensation algorithm of an uncertain biased harmonic disturbance for the linear plant with the input delay, Automation and Remote Control, 2010, vol. 71, N. 8, pp. 1562-1577.

[22] X. Xia, "Global Frequency Estimation Using Adaptive Identifiers," IEEE Transactions on Automatic Control, vol. 47, 1188-1193, 2002. 